\title{
Dificultades del Retorno al trabajo tras Cáncer de Mama
}

\section{Difficulties of Return to Work after Breast Cancer}

\section{Araceli López-Guillén García', José Manuel Vicente Pardo²}

1. Unidad Médica Equipo Valoración Incapacidades. Instituto Nacional de la Seguridad Social. Murcia. España. Cátedra Medicina Evaluadora y Pericial UCAM. España.

2. Unidad Médica Equipo Valoración Incapacidades. Instituto Nacional de la Seguridad Social. Gipuzkoa. España. Cátedra. Medicina Evaluadora y Pericial UCAM. España.

Recibido: 02-01-2020

Aceptado: 12-03-2020

\section{Correspondencia}

Araceli lópez-Guillén García.

Correo electrónico: araceli.lgg@gmail.com

José Manuel Vicente Pardo.

Correo electrónico: josemanuvicente@gmail.com

Resumen

En 2018 se iniciaron en España 12.245 procesos de baja por cáncer de mama, 9.336 alcanzaron los 365 días de duración, se consideró incapacidad permanente en 2.201 trabajadoras. El $76 \%$ de las bajas iniciales por cáncer de mama alcanzan o sobrepasan los 365 días de duración, y de estas bajas de tan larga duración el $18 \%$ pasarán a incapacidad permanente. El cáncer de mama en la mujer en "edad laboral" tiene un pronóstico de supervivencia del $86 \%$, pero tan sólo el $53 \%$ retorna al trabajo de forma efectiva, frente al $47 \%$ que no permanecerá trabajando, bien por declaración de incapacidad permanente, bien por desistimiento no pudiendo rendir en el trabajo, o bien por despido en relación a la pérdida de capacidades, cuando no, por haber pasado a desempleo durante la baja.

Material y método: Revisión bibliográfica en Pubmed, Medline y Scielo hasta mayo de 2019. Estudio de procesos de incapacidad temporal por cáncer de mama en 2018; análisis de 408 procesos de cáncer de mama de duración de incapacidad de más de 365 días, con alta médica laboral y valoración de secuelas; comparación con estudio previo de la IT en 2015.

Conclusiones: El cáncer de mama presenta alto riesgo de exclusión social y laboral, se hace necesario medidas para facilitar el retorno laboral efectivo, reconociendo la "aptitud sobrevenida" de la superviviente al cáncer de mama, que retorna al trabajo. Por ello hay que posibilitar la reintegración laboral progresiva, abordar los factores psicosociales, evitar la controversia entre el alta médica para trabajar y la no aptitud para el trabajo, incentivar a las empresas para la adopción de medidas organizacionales de reinserción, realización del Mapa de Incapacidad para conocer el impacto incapacitante laboral, valorar la incapacidad como indicador de retorno al trabajo, e indicador de salud pública laboral.

Med Segur Trab (Internet). 2020;66(258):47-62

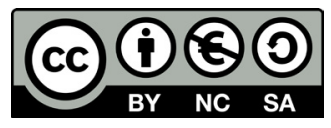

https://creativecommons.org/licenses/by-nc-sa/4.0/ 
Palabras clave: Cáncer de mama. Superviviente de cáncer de mama. Retorno al trabajo. Reinserción laboral. Incapacidad Laboral prolongada por cáncer de mama. Aptitud sobrevenida. Trabajar después del cáncer de mama.

Abstract

From the 12,245 breast cancer withdrawal processes which began in Spain during 2018, 9,336 reached 365 days long and 2,201 workers were considered permanently disabled to work. $76 \%$ of the initial casualties for breast cancer had reached or exceed 365 days and of these long-term sick leave 18\% would have become permanently disabled. Breast cancer in women of „working age“ has a survival prognosis rate of $86 \%$, but only $53 \%$ return to work effectively compared to $47 \%$ who will not continue working. This may be either due to a declaration of permanent disability, or due to withdrawal as not being able to give up on the job, or because of dismissal in relation to the loss of abilities, if not for having gone into unemployment during the sick leave.

Material and Method: Bibliographic review in Pubmed, Medline and Scielo until May 2019. Study of processes of temporary disability due to breast cancer in 2018; analysis of 408 breast cancer processes of disability duration of more than 365 days, with occupational medical discharge and sequel assessment; comparison with the previous study of Temporary Disability in 2015.

Conclusions: Breast cancer presents a high risk of social and occupational exclusion. Measures are needed to ease the effective return of work, recognizing the "survived aptitude" of the breast cancer survivor, who is returning to work. Addressing psychosocial factors, avoiding controversy between medical discharge to work and non-work skills, encouraging companies to adopt organizational reintegration measures, carrying out the Disability Map to know the disabling work impact and assessing the disability as an indicator of return to work and public health at work is therefore necessary for achieving a progressive return to work.

Med Segur Trab (Internet). 2020;66(258):47-62

Keywords: Breast cancer. Breast. Cancer Survivor. Return to work. Job reintegration Prolonged labor disability due to breast cancer. Fitness overtaken. Work after breast cancer. 


\section{ABREVIATURAS}

— INSS Instituto Nacional de la Seguridad Social.

- IT Incapacidad Temporal o "baja”.

- IP Incapacidad Permanente.

- PIT prórroga de incapacidad temporal

— EVI equipo de valoración de incapacidades

\section{INTRODUCCIÓN}

La superviviente al cáncer se enfrenta a un reto añadido "sobrevivir" como trabajadora. El cáncer de mama es un cáncer con pronóstico de supervivencia muy favorable, pero con desfavorable retorno al trabajo ${ }^{1}$. Es preciso facilitar el retorno al trabajo mediante incorporaciones parciales y progresivas, una adaptación efectiva del puesto al retorno al trabajo, ante la "aptitud sobrevenida ${ }^{2}$ ", y un análisis de los factores de riesgo respecto del cáncer de mama en el trabajo.

\section{Análisis de situación}

El cáncer de mama es el segundo tumor más diagnosticado en el mundo y el cuarto en España teniendo en cuenta ambos sexos. En la mujer es el tumor más frecuente. En los años 80 una mujer diagnosticada de cáncer de mama tenían unas posibilidades de estar libre de recaída a los 5 años del diagnóstico en torno al 70 por ciento, en la actualidad estamos cerca del 90 por ciento. Los avances en el diagnóstico precoz y en el tratamiento específico de cada subtipo de tumor mamario han hecho posible esta gran diferencia pronóstica. Incluso en los casos menos favorables, cáncer no curado, podemos hablar de la cronificación del cáncer.

El cáncer de mama tiene un pronóstico de supervivencia muy favorable en torno al $87 \%$ en mujeres en edad laboral, pero sin embargo, el $\mathbf{4 7 \%}$ de las trabajadoras con cáncer de mama no retornarán al trabajo de forma efectiva, bien por pasar a incapacidad permanente laboral, tras la baja, o bien por recaída del tumor, o bien por abandono "voluntario" (desistimiento interior) por ser consciente que no puede responder al trabajo o por priorizar su salud, o de "forma involuntaria u obligada" cuando pasa a desempleo por motivos de salud (ineptitud sobrevenida o no renovación del contrato) lo que supone un alto riesgo de exclusión laboral.

El cáncer de mama figura entre los tres diagnósticos principales de procesos de baja que alcanzan los 365 días, es decir la duración máxima de la incapacidad temporal.

En 2018 de los 12.245 procesos de cáncer de mama que iniciaron la baja (IT), 9.336 alcanzaron los 365 días de duración, el 76,24\%; y por tanto, tan sólo el 23,76\% de las ITs iniciadas por cáncer de mama duraron menos de 365 días.

El cáncer de mama es primera causa de inicio de expedientes de incapacidad permanente a los doce meses, teniendo en cuenta el diagnóstico de "neoplasia maligna de mama mujer".

El cáncer de mama es el primer diagnóstico recogido como "neoplasia maligna de mama mujer” en procesos de IT (baja) que alcanzan los 365 días de duración.

Si bien si agrupamos los "diferentes diagnósticos" por procesos por "cáncer de mama", por "lumbalgias" o por "trastornos de ansiedad", el cáncer de mama ocupa el tercer lugar.

La reincorporación tras cáncer de mama tan sólo se produce en el $53 \%^{1}$, con todo lo que supone para estas mujeres en lo personal y en lo económico no reincorporarse a su trabajo, y el gasto económico en prestaciones y gastos sanitarios para el sistema, por lo que al impacto económico de la incapacidad laboral (prestación económica que compensa la pérdida de la capacidad de ganancia), se suma las derivadas del no retorno efectivo al trabajo. 
El cáncer de mama es causa de largos procesos de baja, por el calendario terapéutico prolongado, por las secuelas del cáncer y por las secuelas de los tratamientos. No olvidemos que algunas de las secuelas aparecen de forma diferida a la conclusión del tratamiento (quimioterapia, radioterapia, y cirugía) y pueden permanecer durante 6 o 12 meses de concluir el mismo. A todo ello añadir que en ocasiones será necesaria, si así lo quiere la mujer, un periodo añadido para la reconstrucción mamaria diferida y la reconstrucción psicológica, en proceso de gran repercusión emocional para la mujer.

Si comparamos estos tiempos obligados por la servidumbre terapéutica que sobrepasan de media los 320 días, con los tiempos "legales" de duración de la baja 365 días, y los 180 máximos de añadido por prorrogar la misma, vemos que el periodo de protección mediante la baja puede resultar escaso para una recuperación completa.

En España se producen ya 27.000 nuevos casos de cáncer de mama año, de los que en su mayoría se producen en plena edad laboral.

La duración "mediana” de las "bajas” (incapacidades temporales) por cáncer de mama es de unos 320 días $^{1}$, estando entre los tres procesos principales que causan "bajas largas" que alcanzan o sobrepasan los 365 días, junto con las lumbalgias y el trastorno ansioso depresivo ${ }^{1}$.

Por otra parte como toda baja larga y máxime en procesos como este, se acrecientan creencias personales sobre si se podrá volver y realizar el trabajo, así como creencias sobre su propia situación personal vital, que a menudo pueblan lo emocional de la paciente de miedos, sentimientos de discapacidad, sentimientos de injusticia, incomprensión y de falta de apoyo del entorno personal, familiar social y laboral. Todo ello dificulta la respuesta a los tratamientos y modifica la actitud que se requiere para ser efectivo en un trabajo.

\section{CÁNCER DE MAMA COMO BAJA PROLONGADA. MATERIAL Y MÉTODO}

Se consultaron los datos de IT 2018 INSS. Se contabilizaron los correspondientes a cáncer de mama en sus diferentes asignaciones diagnósticas, así como el número de días de baja consumidos y su duración media. Así mismo se contabilizaron los procesos de cáncer de mama que duraron 365 días y las resoluciones que en ese momento de establecieron.

A continuación, reportamos algunos datos que confirman al cáncer de mama como un proceso que causa "bajas" (incapacidades temporales) prolongadas y con un alto riesgo incapacitante.

Por los diversos diagnósticos del cáncer de mama se consumieron 2.159.408 días de baja, con una duración media de 176,35 días.

IT por CÁNCER MAMA agrupando los diferentes diagnósticos recogidos como tal

\begin{tabular}{lccc}
\hline \multicolumn{1}{c}{ Diagnóstico } & $\begin{array}{c}\text { Número de } \\
\text { procesos }\end{array}$ & $\begin{array}{c}\text { Número } \\
\text { de días }\end{array}$ & $\begin{array}{c}\text { Duración } \\
\text { media }\end{array}$ \\
\hline N. maligna de mama femenina, sin especif-174.9 & 6.518 & 1.154 .258 & 177,09 \\
\hline Carcinoma in situ de mama-233.0 & 2.882 & 511.503 & 177,48 \\
\hline Neoplasia maligna mama mujer-174 & 2.003 & 365.318 & 182,39 \\
\hline N. naturaleza no especificada de mama-239.3 & 342 & 58.141 & 170,00 \\
\hline Neoplasia evolución incierta de mama-238.3 & 187 & 22.801 & 121,93 \\
\hline Carcinoma in situ mama y aparato genitou-233 & 179 & 29.071 & 162,41 \\
\hline N. maligna de porción central de la mama-174.1 & 99 & 14.151 & 142,94 \\
\hline N. maligna de cola axilar de la mama-174.6 & 26 & 3.140 & 120,77 \\
\hline N. maligna secundaria de mama-198.81 & 9 & 1.025 & 113,89 \\
\hline Todos & $\mathbf{1 2 . 2 4 5}$ & $\mathbf{2 . 1 5 9 . 4 0 8}$ & $\mathbf{1 7 6 , 3 5}$ \\
\hline
\end{tabular}

Datos INSS 2018 
En 2018, se contabilizaron 207.252.343 días de baja (IT) en 5.843.809 procesos iniciados, con una duración media de 35,46 días. Es preciso señalar la trascendencia del cáncer de mama como causante de bajas prolongadas, que se evidencia cuando lo referimos a procesos con duración de más de 365 días. De los 12.245 procesos de cáncer de mama que iniciaron IT, 9.336 alcanzaron los 365 días de duración, el 76,24\% de los procesos de cáncer de mama que causaron baja se prolongaron durante 365 días; y por tanto tan sólo el 23,76\% de las ITs iniciadas por cáncer de mama duraron menos de 365 días.

En los procesos de baja que llegan a 365 días, corresponde al INSS, en ese momento, evaluar si procede la declaración de incapacidad permanente y el grado de la misma, si se considera prorrogar la baja hasta un máximo de 180 días, cuando se prevea va a ver mejoría, o estimar procede el alta médica por curación o mejoría funcional que permite realizar su trabajo.

Resoluciones por cáncer de mama de los 9.336 procesos que alcanzaron los 365 días en 2018

\begin{tabular}{cccc}
\hline Tipo de resolución & Incapacidad Permanente & ALTA & PRÓRROGA de IT \\
\hline Diagnóstico & $\mathbf{N}^{\mathbf{0}}$ de resoluciones & $\mathbf{N}^{\mathbf{0}}$ de resoluciones & $\mathbf{N}^{\mathbf{0}}$ de resoluciones \\
\hline Cáncer de mama & 2.201 & 2.911 & 4.224 \\
\hline$\%$ & $23,57 \%$ & $31,18 \%$ & $45,24 \%$ \\
\hline
\end{tabular}

Datos INSS 2018

Es decir, por los diversos diagnósticos de cáncer de mama, en procesos que alcanzaron los 365 días de duración de la baja, un $23,57 \%$ se estimó la incapacidad permanente en ese momento, un $31,18 \%$ se procedió a considerar el alta médica (mejoría o curación que permite trabajar) y en el $45,24 \%$ se estimó prorrogar la situación de incapacidad temporal. Sobre los procesos con prórroga de la IT antes de los 6 meses ( 545 días desde el inicio de la baja), deberá resolverse si se estima el alta médica, o la incapacidad permanente

El cáncer de mama es la segunda causa de inicio de expedientes de incapacidad permanente de las bajas que alcanzan los doce meses de duración ${ }^{1}$, tras la lumbalgia. Por lo que a pesar de la bonanza en la supervivencia y la mayor eficacia terapéutica, el riesgo de convertirse en incapacidad laboral definitiva es muy alto. El $23,57 \%$ de las bajas que alcanzaron 365 días pasaron a incapacidad permanente en 2018 , en ese momento.

\section{ESTUDIO DE ALTAS MÉDICAS EN SUPERVIVIENTES DE CÁNCER DE MAMA DE PROCESOS DE BAJA QUE ALCANZARON LOS 365 DÍAS}

Para evaluar las dificultades de retorno al trabajo tras cáncer de mama, se hizo seguimiento de $\mathbf{4 0 8}$ procesos de cáncer de mama que duraron $\mathbf{3 6 5}$ días y que fueron dados de alta en el 2018, en los que se recogieron las limitaciones funcionales referidas en el informe médico de valoración previo al pase al Equipo de Valoración de Incapacidades EVI. Es decir se analizaron procesos de supervivientes al cáncer sin consideración de secuelas limitantes incapacitantes para el trabajo.

De los 408 procesos con bajas de más de 365 días de duración, en 82 procesos al alta médica al año de baja se constaba una curación sin secuela alguna, en 326 procesos y se procedió al alta médica laboral con secuelas aunque no incapacitantes. Las secuelas más frecuentes en estas supervivientes al cáncer con alta médica laboral, fueron y por este orden: la astenia o fatiga 68 casos, las parestesias/disestesias (Neurotoxicidad, Síndrome de mano-pie) 60 casos, los trastornos psicológicos (trastorno del ánimo, síntomas depresivos, ansiedad, déficit de memoria, concentración o atención) 58 casos, las artralgias 48 casos, la disfunción de brazo afecto o miembro contiguo 44 casos, el dolor en región paratumoral mamaria 24 casos, el linfedema o edema 16 casos, o que se hallaban pendientes de cirugía de reconstrucción 8 casos. 
De los procesos de cáncer de mama, de duración de la baja superior al año, y que causaron alta médica, un $\mathbf{2 0} \%$ lo fue por curación sin secuelas, un $\mathbf{8 0} \%$ presentaron secuelas aunque no tuvieron alcance incapacitante, es decir, fueron procesos con alta médica por mejoría funcional que permitía retorno al trabajo.

De los casos con alta médica que debían retornar al trabajo y que tenían secuelas

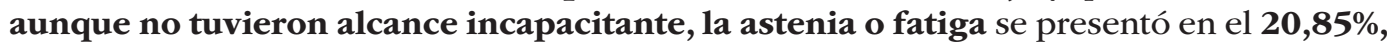
las parestesias/disestesias (Neurotoxicidad, Síndrome de mano-pie) en 18,40\% de los casos, los trastornos psicológicos (trastorno del ánimo, síntomas depresivos, ansiedad, déficit de memoria, concentración o atención) en el $\mathbf{1 7 , 8 0 \%}$ de los casos, las artralgias en el 14,72\% de los casos, la disfunción de brazo afecto o miembro contiguo en el $\mathbf{1 3 , 4 9 \%}$ casos, el dolor en región paratumoral mamaria en el 7,36\% casos, el linfedema o edema en el $\mathbf{4 , 9 0 \%}$ casos, y las que se hallaban pendientes de cirugía de reconstrucción en el $\mathbf{2 , 4 5 \%}$ casos. (Tabla 1)

Tabla 1. Secuelas presentes no incapacitantes al alta médica laboral

\begin{tabular}{lc}
\hline \multicolumn{1}{c}{ SECUELAS PRESENTES NO INCAPACITANTES AL ALTA MÉDICA LABORAL } & $\%$ \\
\hline Astenia o fatiga & $20,85 \%$, \\
\hline Parestesias/disestesias & $18,40 \%$ \\
\hline Trastornos psicológicos & $17,80 \%$ \\
\hline Artralgias & $14,72 \%$ \\
\hline Disfunción de brazo & $13,49 \%$ \\
\hline Dolor & $7,36 \%$ \\
\hline Linfedema & $4,90 \%$ \\
\hline Pendientes de cirugía de reconstrucción & $2,45 \%$ \\
\hline
\end{tabular}

66 procesos con alta recayeron en baja, en los 6 meses siguientes, 16 lo fueron por trastornos psicológicos, 14 por limitaciones en brazo, 12 por astenia fatiga, 10 por artralgias, 8 por neurotoxicidad parestesias disestesias, 6 por cirugía de reconstrucción.

Lo que supone que tras el alta médica laboral de procesos de cáncer de mama con duración de la IT de más de un año, recayeron en baja, en los seis meses siguientes un 16,17\%. Estas recaídas en baja en el periodo de 6 meses siguientes al alta, 25,24\% lo fueron por trastornos psicológicos, $21,21 \%$ por limitaciones en brazo, 18,18\% por astenia fatiga, $15,15 \%$ por artralgias, $12,12 \%$ por neurotoxicidad parestesias disestesias, 9,09\% por cirugía de reconstrucción. (Tabla 2).

Tabla 2. Disfunciones presentes en las recaídas en IT en los 6 meses siguientes al alta

\begin{tabular}{|c|c|}
\hline DISFUNCIONES RECAÍDAS en los 6 meses siguientes al alta médica laboral & $\%$ \\
\hline Trastornos psicológicos & $25,24 \%$ \\
\hline Limitaciones en brazo & $21,21 \%$ \\
\hline Astenia fatiga & $18,18 \%$ \\
\hline Artralgias & $15,15 \%$ \\
\hline Parestesias disestesias & $12,12 \%$ \\
\hline Cirugía de reconstrucción & $9,09 \%$ \\
\hline
\end{tabular}

Es preciso añadir algunas limitaciones a este estudio, a los efectos de valorar un retorno laboral efectivo y duradero, sólo se referencia la consideración de alta médica laboral de procesos que duraron un año o más de baja, se valoran las recaídas en baja, en un plazo muy corto, de tan sólo un máximo de seis meses seguidos al alta, no disponemos de datos sobre si de los casos estudiados hubo consideración de no apto al alta médica, o si hubo desempleo. 


\section{Comparativa de datos con estudio previo 2015}

Estos datos de relevancia del cáncer de mama como proceso principal de larga incapacidad temporal y alto riesgo incapacitante, son similares los de estudio de 2015 del "Comportamiento de la incapacidad temporal de más de 365 días" de López-Guillén García Araceli publicado en Medicina y Seguridad del Trabajo. vol.61) disponible en: http://scielo.isciii.es/scielo.php?script=sci_arttext\&pid=S0465-546X2015000400005\&lng= es\&tlng=es.

Por otra parte añadir que sólo un $\mathbf{5 3 \%}$ de las mujeres retornan de forma efectiva al trabajo tras el cáncer de mama, según datos del trabajo de López-Guillén García, Araceli, \& Vicente Pardo, José Manuel sobre Retorno al trabajo tras cáncer de mama" publicado en Medicina y Seguridad del Trabajo, 63 (246), disponible en: http://scielo. isciii.es/scielo.php?script=sci_arttext\&pid=S0465-546X2017000100051\&lng=es\&tlng=es

Si la supervivencia a los cinco años entre mujeres de 30 a 60 años es del $86 \%$, si una de cada 10 mujeres será diagnosticada de cáncer de mama a lo largo de su vida, si es el tumor más frecuente en las mujeres, si está entre las tres primeras causas de procesos de incapacidad médica larga, si es proceso que se acompaña de riesgo vital, con prolongado calendario terapéutico, si conlleva alta servidumbre terapéutica y de alta intensidad, es proceso que precisa de una especial atención para minimizar su efecto incapacitante laboral, para la mujer trabajadora y para el sistema productivo y prestacional.

Es precisa la elaboración urgente del mapa de incapacidad ${ }^{3}$ que permita conocer procesos incapacitantes, limitaciones que presentan, trabajadores afectados, según tareas ocupaciones, actividad, grupos etarios, circunstancias socioeconómicas. Siendo la incapacidad multifactorial en sus causas y en sus consecuencias, para gestionar adecuadamente la incapacidad no puede ser de otra manera que mediante el análisis de cuantos datos, variables y factores intervienen en su aparición. La incapacidad es un indicador de salud pública, pero también un indicador de resultado ${ }^{4}$. Las dificultades en el retorno al trabajo o el no retorno saludable, efectivo y duradero son consecuencias de situaciones controvertidas entre el alta y el no apto que conviene analizar y más en este proceso de cáncer de mama 5 .

En la dificultad del retorno al trabajo median factores ligados al subtipo de cáncer, su severidad, la intensidad y pauta espaciada de los tratamientos, la evolución individual y respuesta del proceso, la afectación de la calidad de vida, la ausencia prolongada del trabajo, así como otros factores laborales y personales, que todos ellos considerados no siempre hacen que la vuelta al trabajo sea posible ${ }^{1,6,7,8,9,10}$

La trabajadora que ha sobrevenido al cáncer de mama, aunque hoy en día se beneficia de tratamientos más individuales y específicos y menos agresivos, puede haber precisado de cirugía, quimioterapia, radioterapia, hormoterapia, tratamientobiológico, o inmunoterapia, $\mathrm{y}$ aun recuperando las capacidades funcionales puede que permanezca con síntomas y limitaciones difíciles de objetivar y medir como las artralgias, las parestesias, la astenia, la fatiga, el dolor, el deterioro emocional y la merma de la actitud vital, pero evidentemente limitantes. Síntomas que corresponden, por tanto, a procesos difusos pero esperados, como posibles secuelas que es preciso evaluar su carácter limitante.

La situación de alta o mejoría que permite trabajar o la no declaración de incapacidad suponen implícitamente que las capacidades de la trabajadora son compatibles con las capacidades que requiere su trabajo y que el estado de salud, la funcionalidad restante y la continuidad de tratamiento, si fuera el caso, es compatible con el desempeño del trabajo.

El alta médica en procesos de baja que no han superado los 365 días se emite en general por el médico de primaria (salvo muy escasos supuestos en que esta alta es emitida tras control médico por la inspección médica del INSS, o por la inspección médica de las comunidades autónomas). 
En procesos de más de 365 días o en las recaídas tras alta médica del INSS, el alta es sólo emitida por el Equipo de Valoración de Incapacidades del INSS). Así mismo la no declaración de incapacidad al agotar el periodo máximo de 545 días.

\section{DIFICULTADES EN EL RETORNO AL TRABAJO, TRAS CÁNCER DE MAMA}

El camino de retorno al trabajo de las supervivientes al cáncer de mama, no es fácil, la incorporación al trabajo representa para la mujer un punto de realidad contrastada sobre la percepción de su capacitación y creencias vitales antes y después del cáncer. La incorporación al trabajo es volver a un lugar donde se hace visible para las pacientes el nivel de cambio experimentado, la aptitud sobrevenida.

La incorporación obligada de la superviviente al cáncer de mama, bien por no declaración de incapacidad permanente, bien por consideración en revisión de grado de incapacidad permanente concedida de situación de mejora o bien por alta médica de la incapacidad temporal, supone retornar al trabajo tras larga ausencia y sometida a tratamiento agresivo, que aún en este caso favorable, por tratarse de una superviviente no incapacitada laboralmente, determina un retorno laboral con la adquisición de una "aptitud laboral sobrevenida".

La aptitud sobrevenida es la condición de la trabajadora que tras un periodo de incapacidad laboral en que perdió la capacidad para el trabajo, se recuperó esta "capacidad laboral" al alta (no incapacidad, alta médica de la baja), pero se reincorporará al trabajo con una nueva situación de salud (capacidades funcionales) sin restitutio ad integrum, adquiriendo la trabajadora y el trabajo nuevos riesgos para su salud

La "aptitud sobrevenida" derivada de la propia enfermedad, la exposición a tratamientos agresivos durante largo tiempo, y/o las secuelas del propio tratamiento, que hacen que la trabajadora haya adquirido nuevos riesgos para su salud por el propio proceso, y que el trabajo le suponga nuevos riesgos para su salud, por lo que esta aptitud sobrevenida le supone un "sobreriesgo añadido".

Riesgo para su salud laboral derivado de la "aptitud sobrevenida" que es preciso contemplar y aminorar procurando políticas de retorno al trabajo multidisciplinarias que faciliten un retorno laboral eficaz y duradero en el tiempo.

La aptitud sobrevenida deriva de secuelas disfuncionales que permanecen al alta médica de una baja prolongada, y que presuntamente no condicionan la incapacidad permanente, ni justifican continuar en incapacidad temporal. Las disfunciones no incapacitantes laborales, más frecuentes son: la fatiga/astenia, los trastornos psicológicos/ fragilidad psicológica, los trastornos sensitivos disestesias/ parestesias distales, las artralgias, los trastornos de memoria, atención, concentración, el dolor, las disfunciones de órgano y el linfedema. A estos podríamos añadir otros como los trastornos de la piel, trastornos vasculares, trastornos del sueño.

Esta aptitud sobrevenida, sin embargo, tiene un carácter en muchos casos temporal o transitorio, pues las capacidades restantes, presentes a la declaración de alta se entienden son de buen pronóstico evolutivo y no incapacitantes permanentes. En cualquier caso, un periodo de adaptación al inicio de la reincorporación laboral facilitaría a largo plazo el desempeño laboral efectivo y saludable por evolución favorable o por mecanismos de adecuación o confrontación funcional.

El despido objetivo por ineptitud sobrevenida viene regulado legalmente en el Artículo 52. A) del Estatuto de los Trabajadores. La ley prevé la ineptitud sobrevenida como una posible causa de despido objetivo. Por ineptitud, la ley se refiere a la ausencia en la persona del trabajador de las condiciones necesarias para desempeñar su trabajo.

La valoración adecuada de la "Aptitud Sobrevenida", supone que, frente a la "Ineptitud Sobrevenida", que conlleva a la trabajadora que, al perder salud, pierda el trabajo, recupere 
la salud y recupere el trabajo; no obstante, la aptitud sobrevenida, sin adoptar prevención, sin una adecuada adaptación, puede llevar a bajo rendimiento, al presentismo de la trabajadora y en algunos casos abocar en el despido en aplicación de la Ineptitud Sobrevenida.

En el estudio recogido en el apartado 3, de las 326 mujeres que alcanzaron los 365 días de duración de la baja en 2018 y se procedió al alta médica laboral, por entender había curación o mejoría funcional que permitía trabajar y no considerándose por tanto grado de incapacidad permanente laboral, las secuelas más frecuentes en estas supervivientes al cáncer fueron y por este orden: la astenia o fatiga 68 casos, las parestesias/disestesias (Neurotoxicidad, Síndrome de mano-pie) 60 casos, los trastornos psicológicos (trastorno del ánimo, síntomas depresivos, ansiedad, déficit de memoria, concentración o atención) 58 casos, las artralgias 48 casos, la disfunción de brazo afecto o miembro contiguo 44 casos, el dolor en región paratumoral mamaria 24 casos, el linfedema o edema 16 casos, o que se hallaban pendientes de cirugía de reconstrucción 8 casos. Si fueron alta médica quiere decir que se entendía eran compatibles con el tipo de trabajo que desempeñaban.

La incorporación al trabajo tras larga incapacidad supone la exposición a los riesgos presentes en el trabajo y a las condiciones del mismo, así como a la plena disponibilidad y responsabilidad en cuanto al desempeño del trabajo. trabajo.

De las dificultades al retorno efectivo, unas derivan de la trabajadora, otras del

\section{a) Dificultades al retorno al trabajo por parte de la trabajadora.}

Generalmente son incapacidades médicas largas para el trabajo, y por ello suponen para la trabajadora:

- Un aumento del riego de convertirse en incapacidades médicas permanentes, por vencimiento de plazos legales de la incapacidad temporal, por no haber recuperado su funcionalidad para el trabajo, o porque la población trabajadora tras larga incapacidad temporal tienen mayor riesgo de su cronificación incapacitante.

- Un obstáculo a salvar para la trabajadora al cuestionarse ella misma su capacitación para la reincorporación. La trabajadora se pregunta si podrá realizar el trabajo que venía efectuando

- Pueden derivar en la pérdida del empleo o haber derivado durante la situación de baja, máxime en estos tiempos de precariedad y temporalidad de los trabajos.

- Abandono de una situación de "protegida" o "atendida", de la zona de seguridad (de confort), en la que la paciente se veía asistida y protegida, para pasar a la exigencia de su completa autonomía personal.

- Una confrontación entre lo laboral y lo asistencial, cuando se recibe el alta laboral esta no tiene por qué ser equivalente al alta clínica. Puede seguir precisando de controles médicos e incluso de tratamientos, entienden estos no justifican la incapacitación laboral por ser compatibles con el desempeño del trabajo.

- Tiempo de capacitación laboral. Confrontación de plazos

Los plazos legales de la IT y los de recuperación no siempre coinciden.

Plazos legales de incapacidad temporal (IT):

- 365 días de la IT + 180 días de la prórroga= 545 días

- Demora de calificación 180 días + 365 días de la IT + 180 días de la prórroga = 730 días.

Plazos legales de incapacidad permanente (IP):

- Incapacidad permanente revisable, cadencia fijada por el INSS 
- Reserva de puesto de trabajo, con un máximo de 2 años desde la fecha de la incapacidad permanente objeto de revisión.

La estimación de alta médica de la baja supone para la trabajadora la imperiosa necesidad de incorporación al trabajo de hoy para mañana.

\section{b) Dificultades al retorno al trabajo derivadas del trabajo}

Siendo incapacidades médicas largas para el trabajo, suponen desde el entorno del trabajo:

- La consideración de trabajadora sensible. En cualquier caso las limitaciones residuales o el tratamiento que se precisó o el que sigue precisando suponen hacen que la trabajadora ha adquirido un estado de salud con mayor riesgo para el trabajo y un trabajo con mayor riesgo de lesiones o empeoramiento del estado de salud residual.

- No puede establecerse "una” causa del cáncer de mama, sino que debemos considerar diversos factores de riesgo, lo que dificulta la acción preventiva, pues no se ve obligada, para la consideración del alejamiento de un agente concreto.

- No está recogido en España el cáncer de mama como enfermedad profesional (artículo 157 LGSS), ni se referencia en el actual listado de enfermedades profesionales, agente causal vinculado a este proceso; además no es posible su asunción como enfermedad del trabajo (artículo 1562 e LGSS), pues no es enfermedad que podamos relacionar de forma exclusiva con la realización de un trabajo determinado.

- Dicho todo lo cual y a efectos de asociación debemos considera las siguientes sustancias recogidas como cancerígenas:

- Compuestos químicos cancerígenos o genotóxicos (R45/46/49) (H 340, 341, 350 y 351). 1 y 2 A de la IARC.

- Acrilamida, Acrilonitrilos, Agentes anestésicos inhalados, Alcoholes (Epoxi-1propanol), Benceno, Citostáticos, Derivados halogenados de los hidrocarburos alifáticos, saturados o no, cíclicos o no (Cloruro de etileno), Dioxinas, Disruptores endocrinos, Ftalatos. Flexibilizantes del PVC, (Cloruro de Polivinilo), Formaldehído, Hormonas naturales, o idénticas a las naturales o sintéticas, Organoclorados, Óxido de Etileno (ETO), Parabenos, derivados del 4-hidroxibenzoato, Plaguicidas, Policlorobifenilos (PCBs), Sustancias de bajo peso molecular (metales y sus sales, polvos de maderas, productos farmacéuticos, sustancias químico-plásticas, aditivos, etc.), Isoniazida. Subproductos de la incineración de residuos, Sustancias químicas industriales y subproductos industriales: Compuestos de tributilestaño, estanano, etc.), PCB, Policarbonatos, Ftalatos, Cresol, Bisfenol A, Hexaclorobenceno, Estireno, 4-(1,1,3,3-tetrametilbutil)fenol, Fenil-propano, Ignífugos bromados (PBB).

Potencial efecto carcinogénico.

Consultar sustancias en el trabajo "causantes" de cáncer de mama http://risctox. istas.net/abreenlace. asp?idenlace $=6567$

- Se deberá valorar convenientemente, la potencial exposición a:

- Riesgos físicos:

- Radiaciones ionizantes y no ionizantes (campos electromagnéticos) efecto acumulativo tras radioterapia y el propio efecto cancerígeno

- Exposición a condiciones de temperatura extremas (frío o calor) pacientes con procesos secuelares con trastornos de sensibilidad o con linfedema

- Riesgos ergonómicos:

- Las pacientes con linfedema debieran evitar los movimientos repetidos mano-brazo, la sobrecarga de brazo manipulador, el manejo de cargas, 
- Las pacientes con disfunción del hombro evitar actividades de esfuerzo y dinámica intensa con brazo afecto,

- Las pacientes con dolor en brazo o zona pectoral o en el tronco evitar las posturas forzadas, o la manipulación manual de cargas

- Las pacientes con efecto cardiotóxico del tratamiento evitar las actividades de esfuerzo exigente o las actividades con exposición a muy altas temperaturas.

- Caso de las pacientes con cicatrices dolorosas y disfuncionales derivadas de cirugía del cáncer o de la reconstrucción mamaria, evitar el manejo de cargas o las actividades de esfuerzo intenso o continuado

- Las pacientes con cirugía reconstructiva tipo TRAM evitar la prensa abdominal exigente

- Pacientes con astenia tras el tratamiento o artralgias evitar las actividades de esfuerzo exigente

- Riesgos psicosociales:

- Las pacientes con trastornos adaptativos, depresivos o ansiosos evitar las tareas con estrés intenso, y la sobrecarga mental o las actividades con atención y concentración responsable y exigentes, Así mismo valorar efecto de la medicación en manejo de vehículos o herramientas o maquinaria peligrosa.

- Las pacientes con trastornos sensitivos en manos deben evitar el manejo de herramientas de corte o la exposición a temperaturas extremas.

- Las pacientes con linfedema o afectaciones dérmicas (vasculares, infecciosas, cicatriciales) deben evitar las actividades con riesgo de golpes, heridas o quemaduras, o la manipulación de contaminantes biológicos.

- Debe valorarse convenientemente la "turnicidad" como factor "concausal" o de riesgo del cáncer de mama.

Por otra parte no conviene olvidar que la trabajadora retorna al trabajo en una situación no siempre cómoda, pues la empresa se cuestiona si la trabajadora podrá realizar el trabajo y en qué condicionas retornará a su puesto de trabajo. Y siempre se estará pendiente del informe del servicio de prevención y la valoración del reconocimiento de aptitud o los reconocimientos periódicos que se estimen oportunos o la señalización de medidas de vigilancia y de prevención a la trabajadora que retorna a su puesto de trabajo tras larga enfermedad.

También tiene su efecto el componente económico de las largas bajas médicas tras el cáncer de mama que intensifican las consecuencias para la empresa y el sistema productivo. En cuanto a la empresa supone un coste tanto directo por pago de la baja por la empresa $4^{\circ}$ a $15^{\circ} \mathrm{d}$. pago del complemento convenio de IT, coste de la sustitución del trabajador y costes indirectos tiempo perdido por el trabajador ausente, disminución del rendimiento del equipo, pérdida de producción, incapacidad para la sustitución

Pero por encima de cualquier otra consideración a nivel laboral de la trabajadora hay un coste en capacitación, con pérdida de habilidades laborales, o perdida de actualización de conocimientos requeridos para el desempeño del trabajo tras un largo periodo de ausencia del trabajo.

- Permanecer largos periodos de baja supone un riesgo de pérdida del trabajo, bien por haber concluido el contrato laboral en el tiempo que se estuvo de baja, bien por pase a desempleo por ineptitud sobrevenida tras la incorporación al trabajo. 


\section{Consideraciones del alta médica laboral y fin de la prestación económica de la IT}

La declaración del alta médica o "no incapacidad" va a determinar la obligación de reincorporación inmediata al puesto de trabajo (siempre que este exista, pues tras este largo proceso habitual de baja puede que haya finalizado su contrato, o por otras circunstancias pase a desempleo).

Por una parte tras los tratamientos recibidos y las secuelas aunque a la trabajadora se le haya dado el alta (no considerándola incapacitada para el trabajo) adquiere una nueva situación de salud que la hace más vulnerable a los riesgos del trabajo y a su vez el trabajo se torna más peligroso.

Derivado de los tratamientos recibidos y de las secuelas del proceso, aún con recuperación funcional, la superviviente del cáncer de mama ha contraído un mayor riesgo para su salud, pero además al reincorporarse al trabajo, su trabajo presentará mayor riesgo de lesiones y empeoramiento del estado de salud comparativamente con la que tenía antes de contraer la enfermedad constituyendo lo que se denomina aptitud sobrevenida ${ }^{2}$.

En estos casos de la trabajadora que es dada de alta o tras incapacidad se resuelve como no grado de incapacidad por el INSS y debe incorporarse a su trabajo, puede haber problemas bien por no tener trabajo (haber cesado su contrato o haber sido despedida o ser despedida tras la incorporación) o problemas añadidos derivados de la controversia entre esta situación de no incapacidad y una posible declaración de no apta. Lo que supone una incongruencia decisoria entre la declaración de estar capacitada presuntamente para su trabajo y a la par no estar apta (capacitada). Es preciso solucionar esta situación de desamparo de la trabajadora que puede llevarle al paro, con mejora decisoria y arbitraje de esta controversia.

La toma de decisiones también se complica al ser dada de alta y retornar al trabajo, pues la comunicación de información médica entre el ámbito médico sanitario, el de gestión de la incapacidad y el ámbito médico laboral no es siempre completa, sin conocer todas las partes todos los extremos sobre la enfermedad, tratamientos, evolución, capacidad funcional laboral y los requerimientos del trabajo.

Por otra parte en el ámbito sanitario y en parte en el ámbito de gestión de la incapacidad no siempre se conocen las tareas, el trabajo ni la actividad de la empresa, lo que pone en cuestión la decisión de la "capacidad laboral". Estas situaciones podrían mejorarse si contáramos con documentos como la Ficha de Valoración de la Capacidad Laboral aprobada por Osalan en el País Vasco y elaborada desde el Foro de IT de la Academia de Ciencias Médicas de Bilbao y que se expone en documento vinculado a continuación: (http://scielo.isciii.es/pdf/mesetra/v60s1/ponencia16.pdf)

En cuanto a la incorporación inmediata tras el alta añadir a las dificultades al retorno a la situación previa a la enfermedad, la no equiparación a veces del alta médica laboral al alta clínica o sanitaria, a la dificultad de pasar de forma brusca de una situación de protección a la vuelta a la cotidianeidad en todos los ámbitos, pero también en el laboral con exigencia del cien por cien. Por ello el retorno debiera ser en algunos casos paulatino, y progresivo en tiempo y/o en carga de trabajo, para una adaptación correcta y evitar recaídas o presentismo (estar presente en el puesto de trabajo por miedo a perder el empleo, pero sin rendir adecuadamente).

Si admitimos el gran impacto del cáncer en la mujer trabajadora, y las dificultades a su reincorporación al trabajo tal vez debiéramos cuantificar y conocer su verdadera magnitud conociendo que edad tienen las trabajadoras con cáncer de mama, qué trabajo realizan, en qué sectores de la actividad económica, si guarda relación con determinados riesgos, cuántos son los días que se pierden por baja o cuantas las incapacidades permanentes que provocan, la distribución territorial, y otras variables en relación con apoyo familiar y situación económica y laboral. Es decir lo que venimos promoviendo 
como la necesidad urgente de elaborar el Mapa de la Incapacidad. Lo que nos ayudaría a una mejor gestión integral sanitaria, prestacional,laboral y socioeconómica.

El retorno al trabajo es el mejor indicador de salud, más allá de los datos de mortalidad, recurrencia del tumor, morbilidad debemos de analizar si se sucede y en cuánto el retorno al trabajo o el no retorno al trabajo, la incapacidad.

Así las cosas, existen diversos factores que se interactúan en el retorno al trabajo (Figura 1):

Unos derivados del proceso individual y sus consecuencias al finalizar la situación de incapacidad, el subtipo de cáncer, su respuesta al tratamiento, las limitaciones o funcionalidad restante, y la propia precepción de la trabajadora.

Otros factores responden a la actuación de control de la incapacidad, y tiempos legales de la incapacidad.

Por último el retorno laboral efectivo y duradero, responde a factores relacionados con el tipo de trabajo, sector de actividad, tareas, ocupación, riesgos, condiciones especiales y el sistema organizacional de la empresa, así como la actuación obligada preventiva respecto de la consideración de aptitud.

Figura 1. Factores que influyen en el retorno al trabajo

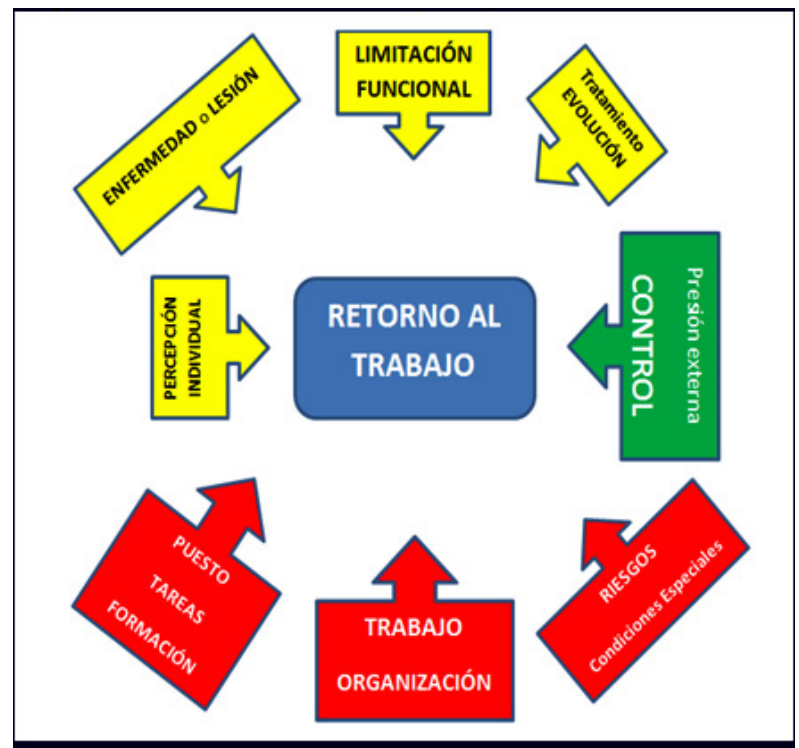

\section{MEDIDAS PARA FACILITAR EL RETORNO AL TRABAJO SALUDABLE $Y$ DURADERO TRAS CÁNCER DE MAMA}

El retorno al trabajo es más complicado cuanto más se prolonga la baja. La posibilidad de reincorporación disminuye tras bajas muy prolongadas, o retorno desde una incapacidad permanente revisada.

El riesgo de pasar al desempleo o quedarse sin trabajo o que el trabajo sea perjudicial para la salud de la trabajadora es importante, en estas situaciones.

\section{PROPUESTAS para un retorno laboral eficaz del trabajador superviviente del cáncer}

- Elaboración del Mapa de Incapacidad Laboral para conocer el verdadero impacto socio laboral del cáncer, y analizar las medidas para fomentar un retorno al trabajo eficaz. 
- Considerar la Incapacidad (el no retorno) como un indicador de salud laboral, un indicador de resultado en salud, un indicador sanitario de calidad en la gestión sanitaria.

- Eliminar las listas de espera, facilitar el acceso público a los tratamientos, eliminando la "toxicidad financiera" derivada de no poder soportar el coste terapéutico. Especial cuidado en la inequidad al acceso a los nuevos tratamientos personalizados de precisión.

- La atención psicológica adecuada, a las supervivientes al cáncer de mama, para identificar y prevenir los procesos psicológicos para mejorar la calidad de vida de las pacientes, y procurar su reintegración a una vida normalizada.

- Elaborar un Plan Estratégico de Protección Integral del Superviviente del Cáncer, para facilitar la reintegración laboral. Pues la capacidad real de trabajar, depende múltiples factores, unos médicos, otros laborales y otros sociales.

- Consideración preventiva de las decisiones del retorno al trabajo, que considere el tránsito o período obligado de adaptación al trabajo tras un alta médica. Garantizando la bioseguridad en lo "laboral".

- Mejora de los sistemas de calificación de la incapacidad/capacidad laboral con una actualización en las consideraciones evaluadoras del cáncer y sus secuelas (Manual de Actuación Médico Evaluadora), un mejor y más exacto conocimiento del trabajo que se realiza, mediante el uso de la Ficha Ocupacional y una reforma del órgano valorador Equipo de Valoración de incapacidades, tanto en su composición, que debe ser fundamentalmente técnica y con representación exclusiva de vocales con conocimientos en medicina evaluadora y salud laboral y sobre conocimientos técnicos del trabajo, requerimientos y condiciones laborales, como en sus competencias, abordando las reclamaciones que sustenten en la controversia entre el no apto y la no incapacidad laboral.

- Mejora de la comunicación entre agentes implicados atención sanitaria, gestores de la incapacidad y el mundo del trabajo, y la toma integral y participativa decisoria entre el ámbito asistencial, el prestacional y el laboral.

- Incorporación parcial al trabajo (Altas Parciales) tras incapacidad. Facilitar la reincorporación parcial, progresiva o gradual, bien en horario, bien en intensidad de las cargas de trabajo, promoviendo una reintegración más efectiva evitando recaídas. En la etapa inicial de retorno facilitar horarios flexibles o reducción horaria que favorezcan la conciliación familiar. Tras un proceso que obligadamente supone una nueva visión vital y un significado nuevo de la importancia y trascendencia del trabajo, con mayor necesidad del apoyo familiar. El retorno gradual al trabajo. El alta parcial para una integración preventiva y efectiva reduciendo las exigencias en tiempo o intensidad para facilitar incorporación. La reintegración paulatina de la trabajadora con "Altas Parciales" facilitaría el reingreso al trabajo y prevendría las recaídas.

- Facilitar la adaptación laboral efectiva ante la "Aptitud sobrevenida", considerando la nueva situación de la trabajadora reincorporada y la presencia de nuevos riesgos contraídos, riesgos en su salud y riesgos del trabajo para su salud. Aún en los casos favorables en los que se valora y se considera el retorno al trabajo de la mujer con cáncer de mama, evaluar la "aptitud sobrevenida" de la trabajadora y los riesgos derivados de la misma.

- Consideración del retorno laboral del superviviente al cáncer como "Trabajadora Sensible".

- Evitar controversias entre alta médica y no apto, yendo más allá de lo prestacional al extender el alta médica, con concordancia decisoria, en ese continuo que debiera existir entre capacidad y aptitud. 
- Mayor detección y protección de los factores psicosociales en el trabajo y riesgos psicosociales, pues una de las secuelas más habituales del superviviente de cáncer es la fragilidad y el deterioro psicológico, que potenciarían el riesgo.

- Mejoras en la organización, condiciones y clima laboral, que fomenten la satisfacción laboral, y el sentimiento de apoyo al retorno. Debe de procederse a potenciar el apoyo del entorno laboral, para facilitar la reincorporación al trabajo y la vigilancia de la salud.

- Incentivación a las empresas para promover el retorno de trabajadores con cáncer. Medidas que supongan beneficios fiscales y reducción de cotizaciones de empresa. Incentivos a las empresas que premien o mejoren el clima de “acogida laboral”.

- Programas Formación y Rehabilitación para potenciar la reinserción laboral del trabajador tras el cáncer.

\section{CONCLUSIONES FINALES. CÓMO PROPICIAR UN MAYOR Y MEJOR RETORNO AL TRABAJO}

Debemos de ser conscientes de la "aptitud sobrevenida" de la mujer superviviente de cáncer de mama. Entendiendo por aptitud sobrevenida la: situación de la trabajadora que tras un periodo de incapacidad en que perdió capacidades, se recuperaron al alta, pero se reincorpora al trabajo con una nueva situación de salud (capacidades) sin restitutio ad integrum, adquiriendo el trabajador y el trabajo nuevos riesgos para su salud.

Hay que facilitar el retorno al trabajo, de la trabajadora con cáncer de mama, en especial de aquellos casos que por el subtipo de cáncer y tratamientos o por la individual evolución y respuesta terapéutica han precisado de largas bajas y ya están curadas o con una mejoría que permite trabajar pero adaptando y acondicionando debidamente el puesto a la trabajadora.

Ante el fenómeno del cáncer de mama y su impacto incapacitante, es preciso conocer el mismo con certeza, por lo que se hace necesario la realización urgente del Mapa de Incapacidad en España ${ }^{3}$, que nos permita conocer a quién afecta la incapacidad, en qué grado y durante cuánto tiempo, grupo etario, sexo, trabajo, tareas, actividad de la empresa, riesgos laborales, situación laboral, zona geográfica y demás variables sociales.

Es imprescindible la mejor y mayor comunicación de aspectos clínicos, laborales y de valoración entre las partes implicadas para la correcta toma de decisiones en gestión de la incapacidad y la valoración de la aptitud laboral. (Ámbito sanitario, ámbito de gestión y ámbito laboral)

El indicador de retorno al trabajo (la incapacidad), es un indicador de calidad de vida, es un indicador de resultado y un indicador de salud pública y laboral ${ }^{4}$. Por lo tanto procede analizar el retorno laboral y sus consecuencias.

Hay que posibilitar el alta parcial para una reintegración laboral progresiva, preventiva y efectiva. Introduciendo medidas de incentivación a las empresas que faciliten tal reinserción y adaptación laboral.

Es preciso abordar los factores psicosociales tanto en lo personal, entorno familiar y social y entorno laboral y organizacional a lo largo del proceso de incapacidad laboral, para favorecer el retorno laboral

Para evitar controversias ${ }^{5}$ entre el alta médica para trabajar y la no aptitud para el trabajo debe de institucionalizarse el uso de la Ficha de Valoración Ocupacional y un procedimiento de arbitraje.

Debe avanzarse en el estudio de los factores que dificultan el retorno incluyendo los factores socioeconómicos, los psicosociales y los relacionados con el tipo de tumor, su 
estadiaje y tratamiento, así como establecer estrategias de adaptación laboral al retorno al trabajo y análisis de las causas que lo dificultan. ${ }^{6,7,8,9,10}$

Así mismo facilitar formación y divulgar la evaluación médico laboral de las situaciones de incapacidad laboral por cáncer de mama ${ }^{11,12}$

\section{REFERENCIAS BIBLIOGRÁFICAS}

1. López-Guillén García Araceli, y Vicente Pardo José Manuel. "Retorno al Trabajo tras Cáncer de Mama". Revista Medicina y Seguridad del Trabajo No 246 páginas 51-67. http://gesdoc.isciii.es/gesdoccontroller?a ction=download\&id=14/07/2017-575fbe0aa1

2. Vicente Pardo JoseMa Ponencia: "La Aptitud Sobrevenida un nuevo concepto preventivo en salud laboral". Jornadas Técnicas de Salud Laboral en las Administraciones Públicas. Sociedad Española de Salud Laboral en la Administración Pública. Marbella 27 octubre 2017.

3. López-Guillén García Araceli. Vicente Pardo, JM. El Mapa de la Incapacidad en España una necesidad urgente. Revista Medicina y Seguridad del Trabajo 2015; 61 (240) 378-392 Escuela Nacional de Medicina del Trabajo Instituto de Salud Carlos III. [ Links ]

4. Vicente Pardo, JM. La Incapacidad laboral como indicador de gestión sanitaria. Revista Medicina y Seguridad del Trabajo vol. 61 N. $^{\circ}$ 239. 2015. [ Links ]

5. Vicente Pardo, José Manuel. No apto pero no incapacitado. La controversia del ser o no ser. Revista Medicina y Seguridad del Trabajo 2017, vol.63, No 247 [citado 2017-11-04], pp.131-158. Disponible en: http://scielo.isciii.es/scielo.php?script=sci_arttext\&pid=S0465-546X2017000200131\&lng=es\&nrm=iso ISSN 1989-7790.

6. Johnsson A, Fornander T, Olsson M, et al. Factors associated with return to work after breast cancer treatment. Acta Oncol. 2007;46(1):90-6.

7. Johnsson A, Fornander T, Rutqvist LE, et al. Predictors of return to work ten months after primary breast cancer surgery. Acta Oncol. 2009;48(1):93-8. doi: 10.1080/02841860802477899.

8. Park, J., \& Shubair, M. (2013). Returning to Work After Breast Cancer: A Critical Review.International Journal of Disability Management, 8. doi:10.1017/idm.2012.7

9. Fantoni S.Q., Peugniez C., Duhamel A., et al. (2010). Factors related to return to work by women with breast cancer in northern France. Journal of Occupational Rehabilitation, 20, 49-58.

10. Vicente Pardo, José Manuel, \& López-Guillén García, Araceli. (2018). Los factores psicosociales como predictores pronósticos de difícil retorno laboral tras incapacidad. Medicina y Seguridad del Trabajo, 64(250), 50-74. Disponible en: http://scielo.isciii.es/scielo.php?script=sci_arttext\&pid=S0465546X2018000100050\&lng=es\&tlng=es.

11. López-Guillén García Araceli, Vicente Pardo, José Manuel. Cáncer de Mama Guía de Valoración de Incapacidad Laboral para Médicos de Atención Primaria. $2^{a}$ edición. Escuela Nacional de Medicina del Trabajo y el Instituto Nacional de la Seguridad Social INSS. 2015. Pág. 79-81. Disponible en: http://www. seg-social.es/Internet_1/LaSeguridadSocial/Publicaciones

12. López-Guillén García A. Vicente Pardo. J. M. Manual de Práctica Clínica 2019 SESPM Sociedad Española de Patología Mamaria. Capítulo 72 Valoración de la Capacidad Laboral en Pacientes con Cáncer de mama. 